\title{
Damping and wave energy dissipation in the interstellar medium
}

\section{Fast magnetosonic waves}

\author{
F. Spanier and R. Schlickeiser
}

\author{
Institut für Theoretische Physik, Lehrstuhl IV: Weltraum- und Astrophysik, Ruhr-Universität Bochum, 44780 Bochum, \\ Germany \\ e-mail: [fspanier;r.schlickeiser]@tp4.rub.de
}

Received 1 March 2004 / Accepted 12 February 2005

\begin{abstract}
The heating rate of the interstellar medium, especially the warm ionized medium, is calculated considering damping of fast magnetosonic waves. Starting with an anisotropic spectrum the energy dissipation rate is derived from the damping rates. The results show that the damping of fast magnetosonic waves is an extraordinarily strong source of heat. The ion-neutral and viscous damping contribute largely to the heating. Furthermore it is shown that one would expect a strong parallelization of the spectrum with exponents well above $\frac{5}{3}$.
\end{abstract}

Key words. magnetohydrodynamics (MHD) - plasmas - turbulence - waves - ISM: general - ISM: magnetic fields

\section{Introduction}

The calculation of interstellar cosmic ray transport parameters, like the parallel and perpendicular spatial diffusion coefficients, the rate of adiabatic deceleration and the momentum diffusion coefficient of cosmic ray particles, requires the knowledge of the power spectra of magnetic and electric fluctuations in the interstellar medium at frequencies below the non-relativistic proton gyrofrequency $\Omega_{\mathrm{i}}$. Adopting the plasma wave viewpoint, in which one models the turbulent electromagnetic fields as a superposition of low-frequency magnetohydrodynamic plasma modes (shear Alfvén waves, fast magnetosonic waves and slow magnetosonic waves), the electric power spectrum is related to the magnetic power spectrum through the Faraday induction law via the dispersion relation of the individual plasma modes.

A problem arises because interstellar magnetic power spectra cannot be measured directly. Only the power spectrum of interstellar electron density fluctuations is accessible via radio scintillation and dispersion measure studies (e.g. Armstrong et al. 1995). Therefore, the relation between magnetic field fluctuations and electron density fluctuations has to be investigated. According to classical MHD theory, shear Alfven waves are incompressible whereas fast magnetosonic waves for lowbeta plasmas exhibit a direct correspondence $\delta n_{\mathrm{e}}^{F} / n_{\mathrm{e}} \simeq \delta B_{z} / B_{0}$ between electron density fluctuations and the fluctuations in the parallel magnetic field. In a full kinetic description of the turbulence (Schlickeiser \& Lerche 2002), shear Alfvén waves become compressible with the wavenumber-dependent relation $\delta n_{\mathrm{e}}^{\mathrm{A}} / n_{\mathrm{e}} \propto\left(k V_{\mathrm{A}} / \Omega_{\mathrm{p}}\right) \delta B_{y} / B_{0}$.

An additional constraint on interstellar magnetic power spectra is provided by considering the heating of the interstellar medium by plasma wave damping. In the first paper of this series, Lerche \& Schlickeiser (2001 - hereafter referred to as Paper I) have calculated the heating rate of the ISM by collisionless Landau damping of fast magnetosonic waves. They demonstrated that the energy loss rate for this process agrees well with the cooling rate of the diffuse interstellar medium if one considers an anisotropic power spectrum and large spatial scales, especially without strong spatial inhomogeneities so that to a first approximation a simple balance of heating and cooling rates

$\epsilon(\rho, T)=\lambda(\rho, T)$

holds. In the second paper of this series, Lazar et al. (2003 hereafter referred to as Paper II) calculated the influence of Alfvén wave damping on plasma heating. Here the authors demonstrated that besides collisionless Landau damping additional damping mechanisms related to gas collisions are important. It is the purpose of the present investigation to consider these additional collisional heating processes also for fast magnetosonic waves because they were not considered in Paper I. As we will show, this modifies the conclusions of Paper I because these additional heating processes are more important than the collisionless Landau damping of fast magnetosonic waves considered there. Throughout this manuscript we use the same notation as in Papers I and II. 
As before, we distinguish between three different phases of the interstellar medium coexisting in pressure equilibrium: cold clouds, a warm intercloud medium (also: warm ionized medium) and hot coronal gas, which is generated by supernova explosions. The temperature of the warm medium can be determined by $21 \mathrm{~cm}$ radio studies. These indicate a temperature range from 6000 to $10^{4} \mathrm{~K}$. Also, from radio studies we can infer the mean HII-density, which is about $0.08 \mathrm{~cm}^{-3}$, and the neutral (HI) hydrogen density that ranges between $0.1-0.2 \mathrm{~cm}^{-3}$. The warm intercloud medium provides the dominant contribution to interstellar electron density fluctuations because its filling factor and its degree of ionization are much higher than that of cold clouds, while the coronal gas, having approximately the same filling factor and degree of ionisation, has a much lower electron density.

As in Paper I we adopt a power spectrum of electron density fluctuation in the form

$P_{n}(\boldsymbol{k})=C_{n}^{2}\left[k_{\|}^{2}+\Lambda k_{\perp}^{2}\right]^{-(2+s) / 2}$.

As mentioned above for fast magnetic waves, magnetic fluctuations are directly proportional to density fluctuations so we can write:

$P_{B}(\boldsymbol{k})=C_{B}^{2}\left[k_{\|}^{2}+\Lambda k_{\perp}^{2}\right]^{-(2+s) / 2}$.

Here $k_{\|}\left(k_{\perp}\right)$ is the component of the wavenumber vector parallel (perpendicular) to the ambient magnetic field; $s$ is the spectral index; $\Lambda$ is the anisotropy parameter. Isotropy corresponds to $\Lambda=1$, whereas if the wave turbulence is aligned more along the lines of thin "platelets" paralleling the ambient field, as suggested by Goldreich \& Sridhar (1995), then $\Lambda \gg 1$. The constant $C_{B}^{2}$ is such that

$\int \mathrm{d}^{3} k P_{B}(\boldsymbol{k})=(\delta B)^{2}$

where $\delta B$ denotes the total magnetic fluctuation. The form of the wave spectrum given by Eq. (3) is assumed to hold between some small wavenumber, $k=k_{\min }$, and a large wavenumber, $k=k_{\max }$, with $k=|\boldsymbol{k}|=\left[k_{\|}^{2}+k_{\perp}^{2}\right]^{1 / 2}$. Spangler (1991) suggests that these wavenumbers are related to outer and inner scale lengths, $l_{\min }$ and $l_{\max }$, respectively, with $l_{\min }=2 \pi / k_{\max }$, $l_{\max }=2 \pi / k_{\min }$. The physical bounds of $l_{\min }$ and $l_{\max }$ are not precisely known, but are probably related to the Whistler wave resonance limit of the interstellar electrons and the physical size of the warm intercloud medium, i.e. the mean cloud distance. The inner scale is estimated by Spangler (1991) to be of the general order of $l_{\min }=10^{7} l_{7} \mathrm{~cm},\left(l_{7}=1\right)$, with the outer scale of order $l_{\max }=10^{17} l_{17} \mathrm{~cm}$ with $l_{17}=1 \mathrm{in}$ hot, ionized regions, and $l_{17}=30$ in the diffuse phase of the interstellar medium. In our study we assume that the power spectrum (3) holds in the warm intercloud medium, which is assumed to be stationary and homogenous.

In this work we use a wavenumber-independent anisotropy factor $\Lambda$, while Goldreich \& Sridhar (1995) predict it to be wavenumber dependent. This approximation is used for two reasons: first, it allows a direct comparison of our calculations with the earlier results of Paper I for fast magnetosonic waves, which were based on the power spectrum (3), and secondly, the mathematically simple form of (3) allows an exact analytical calculation of the wave dissipation rates, which is not possible for more complicated power spectra.

The power spectrum itself results from the balance of all wave damping and driving processes, although a detailed theory and explanation is not available at present. Therefore, our calculations are limited in the sense that we assume a given and fixed power spectrum to determine the heating rate of the interstellar medium, but we do not self-consistently investigate the effect of this energy loss rate on the form of the power spectrum, nor do we investigate the formation of real turbulence spectra in wavenumber space. Although highly needed, such a self-consistent theory lies beyond the scope of the present investigation.

\section{Physical parameters}

For our calculations we describe the warm intercloud medium with the following set of parameters:

$n=0.2 \mathrm{~cm}^{-3}$

$n_{\mathrm{i}}=n_{\mathrm{e}}=0.08 \mathrm{~cm}^{-3}$

$T=T_{\mathrm{e}}=T_{\mathrm{i}}=10^{4} \mathrm{~K}=0.86 \mathrm{eV}$

$v_{\mathrm{i}}=9.1 \times 10^{5} \mathrm{~cm} \mathrm{~s}^{-1}$

$B=4 \mu \mathrm{G}$

$|\delta B|=\sqrt{\delta B^{2}}=0.9 \mu \mathrm{G}$.

The minimum and maximum wavenumbers given by Spangler are

$k_{\min }=\frac{2 \pi}{10^{17}} \mathrm{~cm}^{-1}$

$k_{\max }=\frac{2 \pi}{10^{7}} \mathrm{~cm}^{-1}$.

From these parameters one can derive the following set of additional parameters

a) Ion gyrofrequency

$$
\Omega_{\mathrm{i}}=\frac{e B}{m_{\mathrm{i}} c}=9.58 \times 10^{3} B[\mathrm{G}] \mathrm{Hz}=3.6 \times 10^{-2} \frac{B}{4 \mu \mathrm{G}} .
$$

b) Coulomb logarithm

$$
\begin{aligned}
L \approx & 23.4-1.15 \log n\left[\mathrm{~cm}^{-3}\right] \\
& +3.45 \log T[\mathrm{eV}] \text { for } T<50 \mathrm{eV}=24.0 .
\end{aligned}
$$

c) Ion-ion collision time

$$
\begin{aligned}
\tau_{\mathrm{i}} & =\frac{3 \sqrt{m_{\mathrm{i}}} T_{\mathrm{i}}[\mathrm{eV}]^{3 / 2}}{4 \sqrt{\pi} L e^{4} n_{\mathrm{i}}} \\
& \approx 2.12 \times 10^{7} \frac{T_{\mathrm{i}}[\mathrm{eV}]^{3 / 2}}{n L} \mathrm{~s}=3.5 \times 10^{6} \mathrm{~s} .
\end{aligned}
$$


d) Electron-electron collision time

$$
\begin{aligned}
\tau_{\mathrm{e}} & =\frac{3 \sqrt{m_{\mathrm{e}}} T_{\mathrm{e}}[\mathrm{eV}]^{3 / 2}}{4 \sqrt{2 \pi} L e^{4} n_{\mathrm{i}}} \\
& \approx 3.43 \times 10^{5} \frac{T_{\mathrm{e}}[\mathrm{eV}]^{3 / 2}}{n L} \mathrm{~s}=5.7 \times 10^{4} \mathrm{~s} .
\end{aligned}
$$

e) Alfvén speed

$$
\begin{aligned}
v_{\mathrm{A}} & =\frac{B}{\sqrt{4 \pi m_{\mathrm{i}} n_{\mathrm{i}}}} \\
& \approx 2.18 \times 10^{11} \frac{B[\mathrm{G}]}{\sqrt{n_{\mathrm{i}}\left[\mathrm{cm}^{-3}\right]}}=3.1 \times 10^{6} \mathrm{~cm} \mathrm{~s}^{-1} .
\end{aligned}
$$

\section{Relation between density and magnetic fluctuations}

As we can see from Schlickeiser \& Lerche (2002), we have the following relation between electron density $\left(P_{n n}\right)$ and magnetic fluctuation $\left(P_{B} \simeq P_{z z}\right.$ as the $z$-component of the magnetic field is dominant) spectra for fast magnetosonic waves in a low $\beta$-plasma

$$
\frac{P_{n n}^{\mathrm{M}}(\boldsymbol{k})}{n_{\mathrm{e}}^{2}}=\frac{P_{z z}(\boldsymbol{k})}{B_{0}^{2}} \frac{9}{4}\left(1+\beta \sin ^{2} \theta\right) \simeq \frac{9}{4} \frac{P_{z z}(\boldsymbol{k})}{B_{0}^{2}} .
$$

So for small plasma $\beta$ there holds a direct proportionality between the both spectra. We will only have to normalize our spectrum via the total fluctuating power. In order to derive the constant $C_{B}$ we solve Eq. (4).

$$
\begin{aligned}
\int \mathrm{d}^{3} k P(\boldsymbol{k})= & 4 \pi \int_{k_{\min }}^{k_{\max }} \mathrm{d} k^{2} \int_{0}^{1} \mathrm{~d} k \mathrm{~d} \mu P(k, \mu) \\
= & 4 \pi C_{B}^{2} \frac{k_{\max }^{1-s}-k_{\min }^{1-s}}{1-s} \\
& \times F\left(\frac{1}{2}, 1+\frac{s}{2}, \frac{3}{2}, 1-\frac{1}{\Lambda}\right) \Lambda^{-\frac{2+s}{2}} \\
\Rightarrow C_{B}^{2}= & (\delta B)^{2} \frac{\Lambda^{\frac{2+s}{2}}}{4 \pi \frac{k_{\max }^{1-s}-k_{\min }^{1-s}}{1-s} F\left(\frac{1}{2}, 1+\frac{s}{2}, \frac{3}{2}, 1-\frac{1}{\Lambda}\right)}
\end{aligned}
$$

where $F$ denotes the confluent hypergeometric function ${ }_{2} F_{1}(a, b ; c ; x)$.

\section{Damping rates}

For waves damping at a rate $\gamma_{0}(\boldsymbol{k})$ the energy loss rate $\epsilon_{0}$ is conventionally written in the form (Spangler 1991)

$\epsilon_{0}=\frac{1}{4 \pi} \int \mathrm{d}^{3} k P(\boldsymbol{k}) 2 \gamma_{0}(\boldsymbol{k})$.

The warm phase of the interstellar medium is a partially ionised plasma. The fast magnetosonic waves undergo different types of dissipation in this low-temperature plasma: besides collisionless Landau damping $\left(\gamma_{\mathrm{A}}\right)$ there is damping connected with the collision-effects associated with Joule dissipation $\left(\gamma_{\mathrm{J}}\right)$, ion viscosity $\left(\gamma_{\mathrm{V}}\right)$ (Braginskii 1965; Hollweg 1985) and ion-neutral friction $\left(\gamma_{\mathrm{N}}\right)$ (Kulsrud \& Pearce 1969). All individual damping rates are taken from Tsap (2000). The total damping rate for the fast magnetosonic waves then is

$\gamma_{0}=\gamma_{\mathrm{A}}+\gamma_{\mathrm{J}}+\gamma_{\mathrm{V}}+\gamma_{\mathrm{N}}$

We discuss each contribution in turn. With the respective damping rates $\gamma_{\mathrm{A}}, \gamma_{\mathrm{J}}, \gamma_{\mathrm{V}}$ and $\gamma_{\mathrm{N}}$ it is then straightforward to determine the respective energy loss rates by evaluating the integral of damping rate times wave energy spectrum

$\epsilon_{\mathrm{i}}=C_{B}^{2} \int_{k_{\min }}^{k_{\max }} \mathrm{d} k k^{-s} \int_{-1}^{1} \mathrm{~d} \mu \frac{\gamma_{\mathrm{i}}(\kappa, \mu)}{\left[\mu^{2}+\Lambda\left(1-\mu^{2}\right)\right]^{\frac{2+s}{2}}}$

with $\mu=\cos \theta$. The total energy loss rate is then given by the sum

$\epsilon_{0}=\epsilon_{\mathrm{A}}+\epsilon_{\mathrm{V}+\mathrm{J}}+\epsilon_{\mathrm{N}}$

\subsection{Collisionless Landau damping}

As already described in Paper I the wave damping rate for collisionless Landau damping may be written as

$\gamma_{\mathrm{L}} \simeq\left(\frac{\pi}{8}\right)^{1 / 2} \frac{\sin ^{2} \theta}{\cos \theta} k v_{\mathrm{i}}^{2} v_{\mathrm{e}}^{-1}$.

Compared to the classical formula given by Ginzburg (1961) terms of order $\frac{v_{\mathrm{i}}}{v_{\mathrm{A}}}$ are neglected as the Alfvén speed is much higher than the ion speed in the interstellar setting.

Lerche \& Schlickeiser (2001) found an energy dissipation rate

$$
\begin{gathered}
\epsilon_{\mathrm{L}}=2^{-\frac{5}{2}} \pi^{-\frac{1}{2}} \frac{1-s}{2-s} \frac{k_{\max }^{2-s}-k_{\min }^{2-s}}{k_{\max }{ }^{1-s}-k_{\min }{ }^{1-s}} \\
\times(\delta B)^{2} \frac{v_{\mathrm{i}}^{2}}{v_{\mathrm{e}}} \frac{I\left(\mu_{\mathrm{L}}, \Lambda\right)}{J(\Lambda)} \\
I\left(\mu_{\mathrm{L}}, \Lambda\right)=\int_{\mu_{\mathrm{L}}}^{1} \mathrm{~d} \mu \frac{1-\mu^{2}}{\mu\left(\mu^{2}+\Lambda\left(1-\mu^{2}\right)\right)^{(2+s) / 2}} \\
J(\Lambda)={ }_{2} F_{1}\left(\frac{1}{2}, 1+\frac{s}{2}, \frac{3}{2}, 1-\Lambda^{-1}\right) .
\end{gathered}
$$

The variable $\mu_{\mathrm{L}}$ is the lower limit $\left(\mu_{\mathrm{L}}=\cos \theta \simeq 0.05\right.$ ) of the validity range of the approximation for the Landau damping that we have used in Eq. (26).

There exist approximations for the anisotropy term in the $\operatorname{limit} \Lambda \rightarrow 0$ and $\Lambda \rightarrow \infty$.

$\frac{I\left(\mu_{\mathrm{L}}, \Lambda\right)}{J(\Lambda)}= \begin{cases}\ln \left(\frac{\Lambda}{\mu_{\mathrm{L}}^{2}}\right) ; & \Lambda \ll 1 \\ \frac{s}{\Lambda^{\frac{5}{2}}}\left(\ln \left(\frac{v_{\mathrm{e}}}{V_{\mathrm{A}}}\right)-\frac{1}{2}\right) ; & , \quad \gg 1 .\end{cases}$

The complete derivation can be found in Paper I. Numerical values for our given physical setting are

$\gamma_{\mathrm{L}}=1.32 \times 10^{4} k \frac{\sin ^{2} \theta}{\cos \theta} \mathrm{Hz}$

$\epsilon_{\mathrm{L}}(\Lambda=1)=6.61 \times 10^{-23} \frac{\mathrm{erg}}{\mathrm{cm}^{3} \mathrm{~s}}$. 


\subsection{Joule dissipation}

Tsap (2000) gives the following damping rate for Joule dissipation

$\gamma_{\mathrm{J}}=\frac{1}{2} \frac{m_{\mathrm{e}}}{m_{\mathrm{p}}}\left(\frac{\omega}{\Omega_{\mathrm{i}}}\right)^{2} v_{\mathrm{e}}$

where $v_{\mathrm{e}}$ denotes the electron-electron-collision frequency. Considering the dispersion relation for the fast magnetosonic waves

$\omega^{2}=v_{\mathrm{A}}^{2} \boldsymbol{k}^{2}$,

we can write the damping rate as follows

$\gamma_{J}=\frac{1}{2} \frac{m_{\mathrm{e}}}{m_{\mathrm{p}}}\left(\frac{v_{\mathrm{A}}}{\Omega_{\mathrm{i}}}\right)^{2} v_{\mathrm{e}} k^{2}$.

This corresponds exactly to the damping rate given by Braginskii (1965).

The Joule energy dissipation is then calculated as

$$
\begin{aligned}
\epsilon_{\mathrm{J}}= & \frac{1}{4 \pi} \int \mathrm{d}^{3} k \frac{m_{\mathrm{e}}}{m_{\mathrm{i}}}\left(\frac{v_{\mathrm{A}}}{\Omega_{\mathrm{i}}}\right)^{2} v_{\mathrm{e}} k^{2} P(\boldsymbol{k}) \\
= & C_{B}^{2} \frac{1}{4 \pi} \frac{m_{\mathrm{e}}}{m_{\mathrm{i}}}\left(\frac{v_{\mathrm{A}}}{\Omega_{\mathrm{i}}}\right)^{2} v_{\mathrm{e}} \frac{k_{\max }^{3-s}-k_{\min }^{3-s}}{3-s} \\
& \times 4 \pi \int_{0}^{1} \frac{\mathrm{d} \mu}{\left(\mu^{2}+\Lambda\left(1-\mu^{2}\right)\right)^{1+s / 2}} \\
= & C_{B}^{2} \frac{m_{\mathrm{e}}}{m_{\mathrm{i}}}\left(\frac{v_{\mathrm{A}}}{\Omega_{\mathrm{i}}}\right)^{2} v_{\mathrm{e}} \frac{k_{\max }^{3-s}-k_{\min }^{3-s}}{3-s} \\
& \times \Lambda^{-1-s / 2} F\left(\frac{1}{2}, \frac{2+s}{2}, \frac{3}{2}, \frac{\Lambda-1}{\Lambda}\right) .
\end{aligned}
$$

If we use the value of $C_{B}$ from Eq. (4) we finally find

$\epsilon_{\mathrm{J}}=(\delta B)^{2} \frac{m_{\mathrm{e}}}{m_{\mathrm{i}}}\left(\frac{v_{\mathrm{A}}}{\Omega_{\mathrm{i}}}\right)^{2} v_{\mathrm{e}} \frac{k_{\max }^{3-s}-k_{\min }^{3-s}}{k_{\max }^{1-s}-k_{\min }^{1-s}} \frac{1-s}{3-s}$.

Obviously this result is independent of the anisotropy factor $\Lambda$, which is easy to understand as we actually have no angular dependence of the Joule damping rate (35).

For our given parameters we have the numerical values

$\gamma_{\mathrm{J}}=3.5 \times 10^{7} k^{2} \mathrm{~Hz}$

$\epsilon_{\mathrm{J}}(\Lambda=1)=2.1 \times 10^{-24} \frac{\mathrm{erg}}{\mathrm{cm}^{3} \mathrm{~s}}$.

\subsection{Ion viscosity dissipation}

From Braginskii (1965) we know that viscosity dissipation is given by

$2 \omega \delta_{\mathrm{vis}}=\frac{1}{2 \rho}\left(\left(\frac{\eta_{0}}{3}+\eta_{1}\right) k_{\perp}^{2}+\eta_{2} k_{\|}^{2}\right)$.
So that

$\gamma_{V}=2 \omega \delta_{\text {vis }}$

$$
=\frac{1}{2 m_{\mathrm{i}} n_{\mathrm{i}}}\left(\left(\frac{\eta_{0}}{3}+\eta_{1}\right) \sin ^{2} \theta+\eta_{2} \cos ^{2} \theta\right) k^{2}
$$

where

$\eta_{0}=0.96 n_{\mathrm{i}} \tau_{\mathrm{i}} k_{B} T_{\mathrm{i}}$

$\eta_{1}=0.3 \frac{n_{\mathrm{i}} \tau_{\mathrm{i}} k_{B} T_{\mathrm{i}}}{\left(\Omega_{\mathrm{i}} \tau_{\mathrm{i}}\right)^{2}}$

$\eta_{2}=4 \eta_{1}$

denote the viscosity coefficients for the ions. As $\eta_{1}$ and $\eta_{2}$ scale with $\left(\Omega_{\mathrm{i}} \tau_{\mathrm{i}}\right)^{-2}$ which in the ISM is much smaller than unity $\left(\left(\Omega_{\mathrm{i}} \tau_{\mathrm{i}}\right)^{-2}=1.4 \times 10^{-9}\right)$, at least $\eta_{1}$ can be neglected for $\eta_{0}$. Therefore

$\gamma_{\mathrm{V}}=\frac{1}{6} v_{\mathrm{i}}^{2} \tau_{\mathrm{i}} k^{2}\left(\sin ^{2} \theta+\frac{18}{5}\left(\Omega_{\mathrm{i}} \tau_{\mathrm{i}}\right)^{-2} \cos ^{2} \theta\right)$

where $v_{\mathrm{i}}$ is the ion thermal velocity, which is supposed to be Maxwellian distributed.

This damping rate is now multiplied again with the power spectrum in order to get the energy dissipation rate

$$
\begin{aligned}
\epsilon_{\mathrm{V}}= & \frac{1}{4 \pi} \int \mathrm{d}^{3} k \frac{1}{6} v_{\mathrm{i}}^{2} \tau_{\mathrm{i}} k^{2} \\
& \times\left(\sin ^{2} \theta+\frac{18}{5}\left(\Omega_{\mathrm{i}} \tau_{\mathrm{i}}\right)^{-2} \cos ^{2} \theta\right) 2 P(\boldsymbol{k}) \\
= & \frac{1}{3} v_{\mathrm{i}}^{2} \tau_{\mathrm{i}} \frac{k_{\text {max }}^{3-s}-k_{\min }^{3-s}}{3-s} C_{B}^{2} \\
& \times \int_{0}^{1} \frac{\left(1-\mu^{2}\right)+\frac{18}{5}\left(\Omega_{\mathrm{i}} \tau_{\mathrm{i}}\right)^{-2} \mu^{2}}{\left(\mu^{2}+\Lambda\left(1-\mu^{2}\right)\right)^{(2+s) / 2}} \\
= & \frac{2}{3} C_{B}^{2} v_{\mathrm{i}}^{2} \tau_{\mathrm{i}} \frac{k_{\max }^{3-s}-k_{\min }^{3-s}}{3-s} \\
& \times \Lambda^{-1-s / 2}\left(\left(1-\frac{18}{5}\left(\Omega_{\mathrm{i}} \tau_{\mathrm{i}}\right)^{-2}\right)_{2} F_{1}\left(\frac{3}{2}, \frac{2+s}{2}, \frac{5}{2}, 1-\frac{1}{\Lambda}\right)\right. \\
& \left.-3 F\left(\frac{1}{2}, \frac{2+s}{2}, \frac{3}{2}, 1-\frac{1}{\Lambda}\right)\right) .
\end{aligned}
$$

Again we use Eq. (21) and derive

$$
\begin{aligned}
\epsilon_{V}(\Lambda=1)= & \frac{2}{3}(\delta B)^{2} v_{\mathrm{i}}^{2} \tau_{\mathrm{i}}^{2} \frac{k_{\max }^{3-s}-k_{\min }^{3-s}}{k_{\max }^{1-s}-k_{\min }^{1-s}} \frac{1-s}{3-s} \\
& \times\left(1-\frac{1}{3}\left(1-\frac{18}{5}\left(\Omega_{\mathrm{i}} \tau_{\mathrm{i}}\right)^{-2}\right) \frac{{ }_{2} F_{1}\left(\frac{3}{2}, \frac{2+s}{2}, \frac{5}{2}, 1-\frac{1}{\Lambda}\right)}{{ }_{2} F_{1}\left(\frac{1}{2}, \frac{2+s}{2}, \frac{3}{2}, 1-\frac{1}{\Lambda}\right)}\right)
\end{aligned}
$$

With the given numerical values

$\gamma_{\mathrm{V}}=\left(4.75 \times 10^{17} \sin ^{2} \theta+7.29 \times 10^{7} \cos ^{2} \theta\right) k^{2} \mathrm{~Hz}$

$\epsilon_{\mathrm{V}}(\Lambda=1)=2.29 \times 10^{-14} \frac{\mathrm{erg}}{\mathrm{cm}^{3} \mathrm{~s}}$. 
This dissipation rate (51) for an isotropic spectrum $(\Lambda=1)$ is far too large (15 orders of magnitude) compared to the cooling rate (59), so we have to consider either strong anisotropy or a wave number exponent other than $\frac{5}{3}$ or a different spectral range. We expect to have waves mostly parallel to the ambient magnetic field, as $\epsilon$ is decreasing with increasing $\Lambda$, which means $\Lambda \gg 1$. In Sect. 5 we try to find the exact parameters of the wave spectrum that yield the correct total dissipation rate.

\subsection{Ion-neutral friction}

Kulsrud \& Pearce (1969) have given the following damping rate for ion-neutral collisions

$\gamma_{\mathrm{N}}=\left\{\begin{array}{l}\frac{\omega^{2}}{2 \nu_{\mathrm{N}}} \omega \ll v_{\mathrm{N}} \\ \frac{v_{\mathrm{N}}}{2} \omega \gg v_{\mathrm{N}} .\end{array}\right.$

The ion-neutral collision frequency $v_{\mathrm{N}}$ has an approximate value of $4 \times 10^{-9} n_{\mathrm{H}} \mathrm{Hz}$, so we are in the high frequency limit and can consider the damping rate as an angle-independent constant.

$$
\begin{aligned}
\epsilon_{\mathrm{N}}= & \frac{1}{4 \pi} \int \mathrm{d}^{3} k P(k) 2 \gamma_{\mathrm{N}} \\
= & 2 v_{\mathrm{N}} \int_{k_{\min }}^{k_{\max }} \mathrm{d} k \frac{k^{2}}{k^{2+s}} \int_{0}^{1} \frac{\mathrm{d} \mu}{\left(\mu^{2}+\Lambda\left(1-\mu^{2}\right)\right)^{(2+s) / 2}} \\
= & 2 v_{\mathrm{N}} C_{B}^{2} \frac{k_{\max }^{1-s}-k_{\min }^{1-s}}{1-s} \Lambda^{-(1+s / 2)} \\
& \times F\left(\frac{1}{2}, \frac{2+s}{2}, \frac{3}{2}, 1-\frac{1}{\Lambda}\right) .
\end{aligned}
$$

We note that this rate depends only on the total strength of the magnetic turbulence and not on the extent of the turbulence spectrum. After inserting Eq. (21) we have the simple and $\Lambda$-indepedent expression

$\epsilon_{\mathrm{N}}=\frac{\nu_{\mathrm{N}}}{4 \pi}(\delta B)^{2}$.

The numerical values are given by

$\gamma_{\mathrm{N}}=4 \times 10^{-10} \mathrm{~Hz}$

$\epsilon_{\mathrm{N}}=5.15 \times 10^{-23} \frac{\mathrm{erg}}{\mathrm{cm}^{3} \mathrm{~s}}$.

This dissipation rate is also two orders of magnitude too high compared to the cooling rate, indepedent of the form of the spectrum. To change the magnitude of ion-neutral heating, the total power of fluctuations has to be changed.

The dissipation rates for all damping processes are shown in Fig. 1.

\section{Understanding the wave power spectrum}

From Minter \& Spangler (1997) we know that the cooling rate of the diffuse interstellar medium is

$L_{\mathrm{R}}=5 \times 10^{-24} n_{\mathrm{e}}^{2} \mathrm{erg} \mathrm{s}^{-1} \mathrm{~cm}^{-3}$

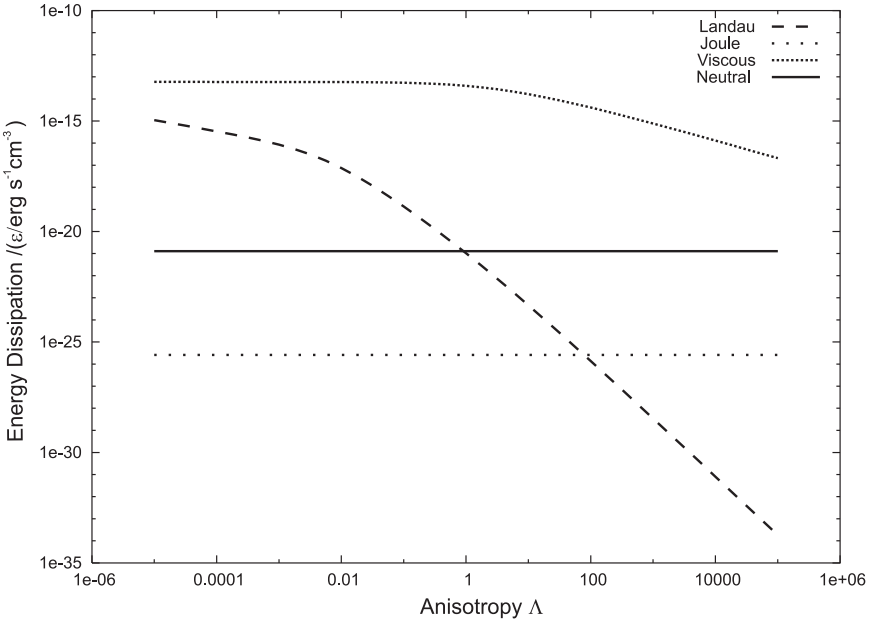

Fig. 1. Anisotropy dependence of different energy dissipation processes.

with the electron density of the interstellar medium of $n_{\mathrm{e}}=$ $0.08 \mathrm{~cm}^{-3}$ we have a cooling rate of $3.2 \times 10^{-26} \mathrm{erg} \mathrm{s}^{-1} \mathrm{~cm}^{-3}$. Obviously, our heating rates (compare Eqs. (51) and (58) with Eq. (59)) are much higher than the cooling rate if we adopt Kolmogorov scaling $(s=5 / 3)$ and an isotropic spectrum $(\Lambda=1)$.

Within the current hypothesis of a steady-state configuration of heating and cooling, in order to achieve agreement of the wave turbulence spectrum with the constraints from the cooling rate we have two options: we can either modify

1. the parameters $s$ (spectral index) and $\Lambda$ (anisotropy) of our turbulence spectrum or

2. the maximum wavenumber $k_{\max }$ of the fast magnetosonic waves because an early cutoff of the spectrum can be expected with the dominant damping process (viscous damping) scaling as $\propto k^{2}$ (see Eq. (41)).

First we consider different cases for which we calculate the spectral index $s$ starting with an isotropic spectrum, and then we gradually increase the parameter $\Lambda$ with the limit of a fully parallel spectrum.

As the ion-neutral damping is only determined by the collision frequency between ions and neutrals and the fluctuating magnetic power $\delta B_{\mathrm{M}}$, the heaiting rate by ion-neutral friction can be used to fit the fluctuating magnetic power as it is the only parameter here. We attribute $50 \%$ of the heating to ion-neutral damping, i.e. $\epsilon_{\mathrm{N}}=0.5 L_{\mathrm{R}}$, so that Eqs. (58) and (59) yield

$4 \times 10^{-9} n_{\mathrm{H}}\left(\delta B_{\mathrm{M}}\right)^{2} \frac{1}{2 \pi}=5 \times 10^{-24} n_{\mathrm{e}}^{2}$

$\delta B_{\mathrm{M}}=1.59 \times 10^{-8} \mathrm{G}$

Although this is a very small fluctuation power, it seems to be a plausible starting point. Fast magnetosonic waves are strongly damped, so that energy in that kind of wave dissipates very fast, leading to the fact, that there cannot be much fluctuation energy stored in the fast magnetosonic waces. This conclusion is in contradiction to Paper I, where only collisionless Landau damping was considered. The argument is still the same; in 
Paper I the damping rate almost fitted the cooling rate, but our new results contradict that earlier result: for FMS-dominated turbulence, heating and cooling are not in balance.

\subsection{Isotropic spectrum with steep power law spectral index $s$}

As mentioned above we have fixed the value of $\delta B_{\mathrm{M}}$ by demanding

$\epsilon_{\mathrm{N}}=\frac{1}{2} L_{\mathrm{R}}$

The remaining parameters $s$ and $k_{\max }$ may be calculated by attributing the remaining $50 \%$ heating to viscous damping, i.e.

$\epsilon_{\mathrm{V}}=\frac{1}{2} L_{\mathrm{R}}$

We leave the parameter $k_{\min }$ unchanged as it has only a minor influence on the total dissipation rate. We will determine the values of $s$ and $k_{\max }$ in the following way: we will assume an isotropic spectrum $\Lambda=1$ keeping the value for $k_{\max }$ fixed (with the value given by Spangler 1991) to calculate $s$. In a second calculation we will do this vice versa assuming a Kolmogorovlike spectrum $(s=5 / 3)$ to calculate $k_{\max }$. In the end we will derive the general relation between $s$ and $k_{\max }$ to identify all possible parameter sets for isotropic spectra.

The first method of fixing $k_{\max }$ and varying $s$ yields from

$\epsilon_{\mathrm{V}}(\Lambda=1)=\frac{1}{2} L_{\mathrm{R}}=\epsilon_{\mathrm{N}}$

the value $s \approx 2.4$.

This result shows that we have to assume a very high spectral index $s$ (much higher than the Kolmogorov value 5/3) and very low fluctuating power to justify the chosen $k_{\min }$ and $k_{\max }$ of Spangler.

The second modification of fixing $s=5 / 3$ and varying $k_{\max }$ yields from Eq. (64) $k_{\max }=1.38 \times 10^{-12} \mathrm{~cm}^{-1}$, which is five orders of magnitude smaller than the values of Spangler.

Varying both the spectral index $s$ and $k_{\max }$ yields the relation

$\frac{s-1}{3-s} k_{\max }^{3-s} k_{\min }^{s-1}=\frac{9 v_{\mathrm{N}} m_{\mathrm{i}} n_{\mathrm{i}}}{2 \eta_{0}}$

in the approximation $k_{\max } \gg k_{\min }$ from Eq. (64). For given numerical values and different values of $s$ we calculate the wavenumber of the wave spectrum cutoff. The results are shown in Fig. 2.

\subsection{Fully parallel spectrum}

As Fig. 1 shows, a small value of $\Lambda \ll 1$ does not modify the viscous damping rate. However, large values of $\Lambda \gg 1$ have a strong influence on the damping. Therefore, only for values of $\Lambda \gg 10^{8}$ giving a fully parallel spectrum are we able to justify $s=5 / 3$.

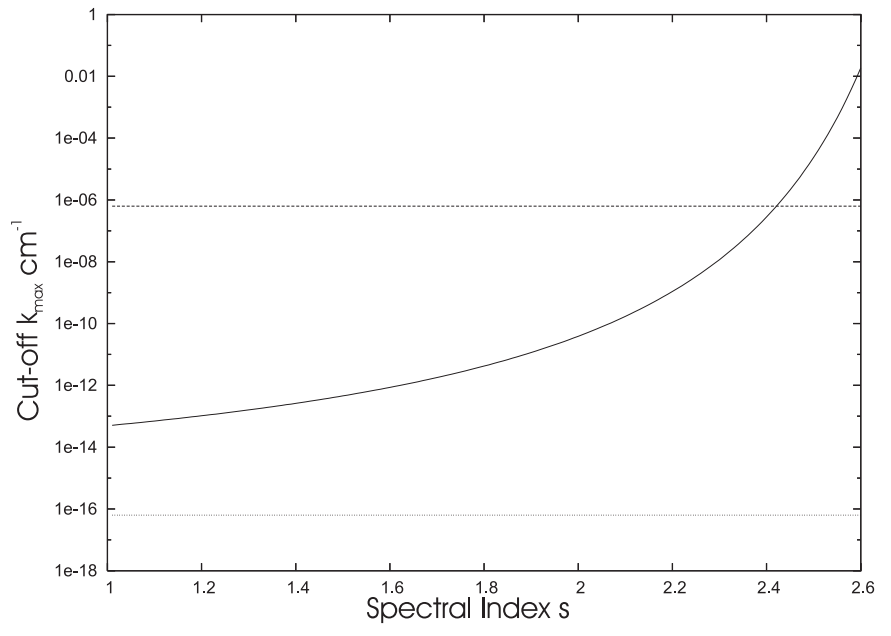

Fig. 2. Relation between cutoff wavenumber $k_{\max }$ and spectral index $s$ for isotropic turbulence. The dotted line shows Spangler's $k_{\min }$ while the dashed shows his $k_{\max }$.

\section{Discussion and summary}

We have calculated the main damping processes of fast magnetosonic waves in the interstellar medium. Using numerical parameters derived from observations we found that for isotropic turbulence ion viscosity and ion-neutral damping in particular lead to a much higher energy dissipation than the cooling rate allows in a steady state case. This leads to two conclusions:

1. Fluctuations of the ISM consist predominantly of shear Alfvén waves. Fast magnetosonic waves are damped instantaneously, so that the power ratio $\left(\propto(\delta B)^{2}\right)$ of fast magnetosonic waves to Alfvén waves is well below $0.1 \%$

2. The assumed standard wave spectrum (3) implies either

(a) values of $\Lambda \gg 10^{8}$ for $s=5 / 3$ (i.e. extremely parallel fast magnetosonic turbulence);

(b) steep spectral indices $s>2.4$ for isotropic turbulence and perpendicular turbulence;

(c) the existence of a high wavenumber cutoff for isotropic turbulence with a spectral index $s=5 / 3$, five orders below the standard cutoff attributed to gyroresonant damping.

The main question arising from our results is the physical plausibility of the proposed modifications (steeper spectra, low cutoff, parallel spectra). The main issue of all three modifications proposed above is the very small fluctuating power of fast magnetosonic waves, which is a result quite different from Paper I. Observational results cannot clarify this point as they do not allow us to distinguish between different wave types in the ISM.

We will initially analyze the energy conservation in the ISM turbulence to justify our model. Our basic assumption was the steady state model

$$
\begin{aligned}
& E_{\mathrm{tot}}=\frac{(\delta B)^{2}}{8 \pi}=\text { const. } \\
& \dot{E}_{\mathrm{tot}}=P_{\text {gain }}-P_{\text {loss }}=0 \\
& \Rightarrow P_{\text {gain }}=P_{\text {loss }}
\end{aligned}
$$


As the outgoing power $\left(P_{\text {loss }}\right)$ has clearly been identified as the plasma wave damping, we will now try to justify supernovae as the main input $\left(P_{\text {gain }}\right)$ process.

As a simplified model we assume a cylindrical galaxy $(r=$ $50000 \mathrm{Ly}, h=3000 \mathrm{Ly}$ ) completely filled with diffuse ionized gas, which is heated by supernovae (the typical energy output for supernovae is $E=10^{51} \mathrm{erg}$ and the mean time between two supernovae is approx. $\tau_{\mathrm{SN}}=10^{9} \mathrm{~s}=30$ years).

First, we calculate the average input power from supernovae for the total Galaxy

$P_{\mathrm{SN}}=\frac{E_{\mathrm{SN}}}{\tau_{\mathrm{SN}}}=10^{42} \frac{\mathrm{erg}}{\mathrm{s}}$.

This is in reasonable agreement with the total cooling rate

$P_{\text {cool }}=L_{\mathrm{R}} V=7 \times 10^{41} \frac{\mathrm{erg}}{\mathrm{s}}$,

which is $70 \%$ of the input supernova power.

The energy of the supernovae is approximately sufficient to sustain the energy balance. But still one question remains: is it likely that such a high fraction of the heating power from the supernovae is ultimately dissipated by ISM heating, probably via the formation of supernova shock waves, which are important for the generation of turbulence? Thus the input power is almost fully available as wave energy. On the other hand, the alternative energy-consuming process, which is according to Schlickeiser (2002) the acceleration of cosmic rays, only needs an average power of $10^{40} \mathrm{erg} / \mathrm{s}$. As it seems there are no other processes in an interstellar phase at rest (no convective motions like galactic winds) which could possibly consume the available energy and as the input power and the heating fit remarkably well (though we used a very rough model) it is reasonable to argue that ISM heating is a stationary process driven by supernovae. It should be noted, however, that the given figures are only rough approximations, the supernova-frequency especially cannot be treated as a constant and a strong deviation from the average would lead to a cooling of the ISM.

One may also derive time scales from the total energy $E$ instead of the power $P=\dot{E}$, but these time scales are not suitable for the dynamical thermal equilibrium, which we examine, as there is no intrinsic relation between $E$ and $\dot{E}$. Those time scales can only give estimates for the time the turbulent field would need to build or decay when either the input or output power would be turned off.

The "decay time scale" is the ratio of field energy and loss power (the plasma wave heating rate).

$\tau_{\text {decay }}=\frac{(\delta B)^{2}}{8 \pi L_{R}}$.

We evaluate this time scale for both fast magnetosonic and Alfvén waves.

$\tau_{\text {decay }}(\mathrm{AW})=1 \times 10^{12} \mathrm{~s}=3 \times 10^{4}$ years

$\tau_{\text {decay }}(\mathrm{FM})=3 \times 10^{8} \mathrm{~s}=10$ years.

These results show that Alfvénic turbulence would persist much longer than fast mode waves after turning off supernovae, but it also shows that the decay of energy in fast magnetosonic waves is three times shorter than the typical input rate from supernovae $\left(\tau_{\mathrm{SN}}=10^{9} \mathrm{~s}\right)$.

This could mean that fast magnetosonic waves can only be found in the vicinity of supernovae and are damped completely after a supernova occurs.

Another possibility is the nonlinear interaction of Alfvén waves as a generation mechanism for the fast magnetosonic waves (see e.g. Chin \& Wentzel 1972). Particularly the production rate of fast waves by Alfvén waves could help our understanding of the energy transfer in the ISM.

Also the time scale of field creation should be analyzed. This time scale is derived from turbulent electromagnetic energy and input power. Again we differentiate between Alfvén and fast magnetosonic waves. These "rise time scales" are given by

$\tau_{\text {rise }}=\frac{(\delta B)^{2}}{8 \pi P_{\mathrm{SN}}}$

$\tau_{\text {rise }}(\mathrm{AW}) \simeq \tau=7.5 \times 10^{11} \mathrm{~s}=2.5 \times 10^{4}$ years

$\tau_{\text {rise }}(\mathrm{FM})=1.5 \times 10^{9} \mathrm{~s}=5 \times 10^{1}$ years.

These timescales differ by nearly three orders of magnitude, which may support the physical model that the two wave types have different origins. The fast magnetosonic wave timescale is again of the order of $\tau_{\mathrm{SN}}$ while Alfvénic turbulence needs much longer to develop. It should be noted, however, that the order of $\tau_{\text {rise }}(\mathrm{AW})$ is not in contradiction to our assumption of supernova heating. If we start in a cold, not ionized gas there is no cooling function $L_{\mathrm{R}}$ (which is proportional to the electron density) so that in the beginning exponential heating of the ISM is possible.

The next point concerning the physical plausibility is the possibility of a nearly parallel spectrum. For Alfvén waves this matter has been addressed by Goldreich \& Sridhar (1995), but their (disputed) process is not applicable to fast magnetosonic waves. Nonetheless the ISM is marked by strong anisotropies, which may result in anisotropic spectra. Again further insight into the production processes of FMS waves would be helpful, but in contrast to the question of input power of the spectrum, the anisotropy may only be explained by the damping itself. If we imagine an isotropic input spectrum, we may see after short times that with increasing wavenumber the anisotropy increases, as the damping of perpendicular waves is dominant. This may be modelled by wavenumber dependent anisotropy factors $\Lambda$, which for reasons of simplicity are not included in our model.

The last point to be discussed is the possibility of very steep spectra. If we stick to the Kolmogorov (1941) or Kraichnan-Iroshnikov (1965) theory we should expect a 5/3 or $3 / 2$ spectrum, but one point in the derivation of those theories seems not to be fulfilled in our case: The K41 (i.e. turbulence with an inertial range power law $s=5 / 3$ ) and KI (i.e. $s=3 / 2$ ) theories consider an inertial range, where the form of the turbulence spectrum is given by the local transport in wavenumber space, with damping being neglected in that range. But as we already pointed out, the ISM has strong damping features. This does not mean that there is no inertial range but there 
could be additional effects due to the dispersion range studied by Stawicki et al. (2001), an intermediate regime between the inertial and dissipation range. Thus our findings are not in contradiction to Kolmogorov theory but they seem to indicate that the inertial range of the ISM turbulence is negligible.

Acknowledgements. We thank the anonymous referee for the very constructive and helpful report. We acknowledge partial support by the Deutsche Forschungsgemeinschaft through Sonderforschungsbereich 591. We would also like to thank Urs Schaefer-Rolffs for providing the figures.

\section{References}

Abramowitz, M., \& Stegun, I. A. 1972, Handbook of Mathematical Functions, National Bureau of Standards, Washington

Armstrong, J. W., Rickett, B. J., \& Spangler, S. R. 1995, ApJ, 443, 209

Braginskii, S. I. 1965, Rev. Plasma Phys., 1, 205
Chin, Y., \& Wentzel, D. G. 1972, Ap\&SS, 16, 465

Gary, S. P. 1986, J. Plasma Phys., 35, 431

Ginzburg, V. I. 1961, Propagation of Electromagnetic Waves in Plasma (New York: Pergamon Press)

Goldreich, P., \& Sridhar, S. 1995, ApJ, 438, 763

Hollweg, J. V. 1985, J. Geophys. Res., 90, 7620

Kolmogorov, A. N. 1941, Dokl. Akad. Nauk. SSSR, 30, 301

Kraichnan, R. 1965, Phys. Fluids, 8, 1835

Kulsrud, R. M., \& Pearce, W. P. 1969, ApJ, 156, 445

Lazar, M., Spanier, F., \& Schlickeiser, R. 2003, A\&A, 410, 415 (Paper II)

Lerche, I., \& Schlickeiser, R. 2001, A\&A, 366, 1008 (Paper I)

Lerche, I., \& Schlickeiser, R. 1982, MNRAS, 201, 1041

Minter, A. H., \& Spangler, S. R. 1997, ApJ, 485, 182

Schlickeiser, R., \& Lerche, I. 2002, J. Plasma Phys., 68, 191

Schlickeiser, R. 2002, Cosmic Ray Astrophysics (Berlin: Springer) Spangler, S. R. 1991, ApJ, 376, 540

Stawicki, O., Gary, S. P., \& Li, H. 2001, JGR, 106, 8273

Tsap, Y. T. 2000, Sol. Phys., 194, 131

Sturrock, P. A. 1994, Plasma Physics (Cambridge University Press) 